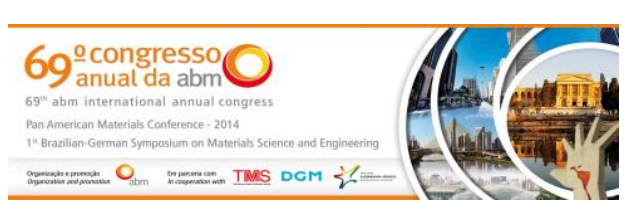

Tema: Metalurgia física e comportamento de materiais em temperaturas elevadas

\title{
USO DE TRANSFORMADOR COM NÚCLEO DE LIGA AMORFA EM AERONAVES*
}

\author{
Wagner Angelo da Silva ${ }^{1}$ \\ Osmar Ogashawara ${ }^{2}$ \\ Alberto Moreira Jorge Junior ${ }^{3}$
}

\section{Resumo}

A crescente demanda por energia elétrica nas aeronaves modernas pode ser constatada pelo aumento da potência elétrica gerada a bordo, como exemplo o Boeing 787 produz 1000kVA, 200V trifásico, $400 \mathrm{~Hz}$ variável contra 240kVA, 200V trifásico, $400 \mathrm{~Hz}$ fixo produzido pelo Airbus A330, apesar de possuírem aproximadamente as mesmas dimensões. Devido à necessidade de redução de massa de transformadores, a frequência utilizada na geração de eletricidade na aeronave é de $400 \mathrm{~Hz}$ o que acaba produzindo um aumento das perdas em núcleos de $\mathrm{FeSi}$ destes transformadores, principalmente pelo aumento das correntes parasitas no núcleo. Transformadores elétricos com núcleos de ligas amorfas a base de Fe vêm apresentando redução de até $70 \%$ das perdas no núcleo quando operados em $50 \mathrm{~Hz}$ ou $60 \mathrm{~Hz}$. Assim, esse estudo visa discutir as vantagens e desvantagens do uso de transformadores elétricos com núcleos de ligas amorfas a base de Fe em aeronaves. Os resultados mostram que, com o desenvolvimento de novas ligas, tal utilização mostra-se viável devido a uma redução de peso de tais transformadores.

Palavras-chave: Transformador; Liga amorfa; Aeronave.

\section{USING AMORPHOUS ALLOYS IN CORES OF ONBOARD POWER ELECTRICAL TRANSFORMERS OF AIRCRAFTS}

\section{Abstract}

The increasing demanding for electrical energy onboard of modern aircrafts can be noticed by the increase of the electrical power generation, as for example: Boeing 787 generates 1000kVA while Airbus A330 generates 240kVA, although they have almost the same size. $400 \mathrm{~Hz}$ alternate current is used for mass reduction in transformers and generators onboard, but this higher frequency also increases the FeSi core transformer losses, mainly by the core eddy currents. Core transformers of amorphous alloys have been proved to reduce the loss in almost $70 \%$ when operating at $50 \mathrm{~Hz}$ or $60 \mathrm{~Hz}$. Thus, this study aims to discuss the advantages and disadvantages of using cores of Fe-based amorphous alloys in onboard electric transformers. The results show that with the development of new alloys, such use appears to be feasible due to a reduction in weight of such transformers.

Keywords: Transformer; Amorphous alloy; Aircraft.

\footnotetext{
Engenharia Elétrica, Engenheiro, Mestrando, PPGCEM, UFSCar, São Carlos, SP, Brasil.

Engenharia Elétrica, Doutor, Professor, DEE, UFSCar, São Carlos, SP, Brasil.

Engenharia Elétrica, Doutor, Professor, LCE, UFSCar, São Carlos, SP, Brasil.
}

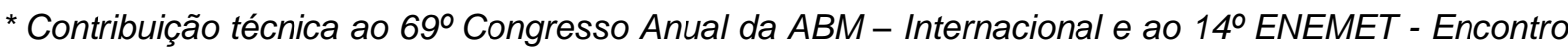
Nacional de Estudantes de Engenharia Metalúrgica, de Materiais e de Minas, 21 a 25 de julho de 2014, São Paulo, SP, Brasil.
} 


\section{INTRODUÇÃO}

O aumento da demanda por eletricidade nas aeronaves modernas é consequência da substituição de sistemas hidráulicos, pneumáticos e mecânicos por sistemas elétricos com mesma funcionalidade. Como exemplos pode-se citar a utilização de freios acionados por motores elétricos ao invés de freios acionados por atuadores hidráulicos; sistema de pressurização da cabine que utiliza ar do exterior bombeado por motores elétricos em substituição do ar pressurizado sangrado dos motores a combustão da aeronave; o uso de janelas com material que altera sua transparência eletricamente permitindo maior ou menor passagem de luz do ambiente exterior eliminando os tampões plásticos acionados mecanicamente [1]. Pode-se também observar o aumento do consumo de eletricidade ao se comparar o total de potência produzida pelos geradores elétricos acoplados aos dois motores, aeronaves tradicionais de mesmo porte do Boeing 787 geram até 240kVA enquanto a potência gerada em um Boeing 787 é de até 1000kVA [2].

Tais substituições também visam redução peso, pois, em uma primeira aproximação, considera-se a quantidade de combustível consumida no voo proporcional ao peso da aeronave, uma vez que o aumento de peso aumenta o arrasto [3].

Após a segunda guerra mundial, muitos fabricantes de aeronaves civis adotaram a frequência de $400 \mathrm{~Hz}$ para a geração de eletricidade a bordo, buscando diminuir o peso dos dispositivos elétricos que operam em corrente alternada. Tal redução pode ser comprovada pela Lei da Indução de Faraday, onde a massa do núcleo de um transformador e a quantidade de espiras de fio de cobre podem ser diminuídas com o aumento da frequência, mantendo-se a tensão induzida constante. Infelizmente, o aumento na frequência resulta também em aumento de perdas no núcleo. A perda por histerese é proporcional à frequência de operação enquanto a perda por correntes parasitas é proporcional ao quadrado da frequência. Considerando-se a potência produzida nas aeronaves tradicionais, tais perdas são aceitáveis. Entretanto, ao serem consideradas potências mais elevadas, há a necessidade de opções para a redução de tais perdas.

Núcleos de transformadores feitos com lâminas de liga amorfa de FeBSi apresentam redução de perdas em aproximadamente $70 \%$ em comparação ao FeSi cristalino, quando operados em $50 \mathrm{~Hz}$ ou $60 \mathrm{~Hz}$. Conforme mostrado na Figura 1, nota-se a maior temperatura no núcleo de FeSi cristalino, à esquerda, devido às suas maiores perdas em comparação ao núcleo de FeBSi amorfo [4], à direita.

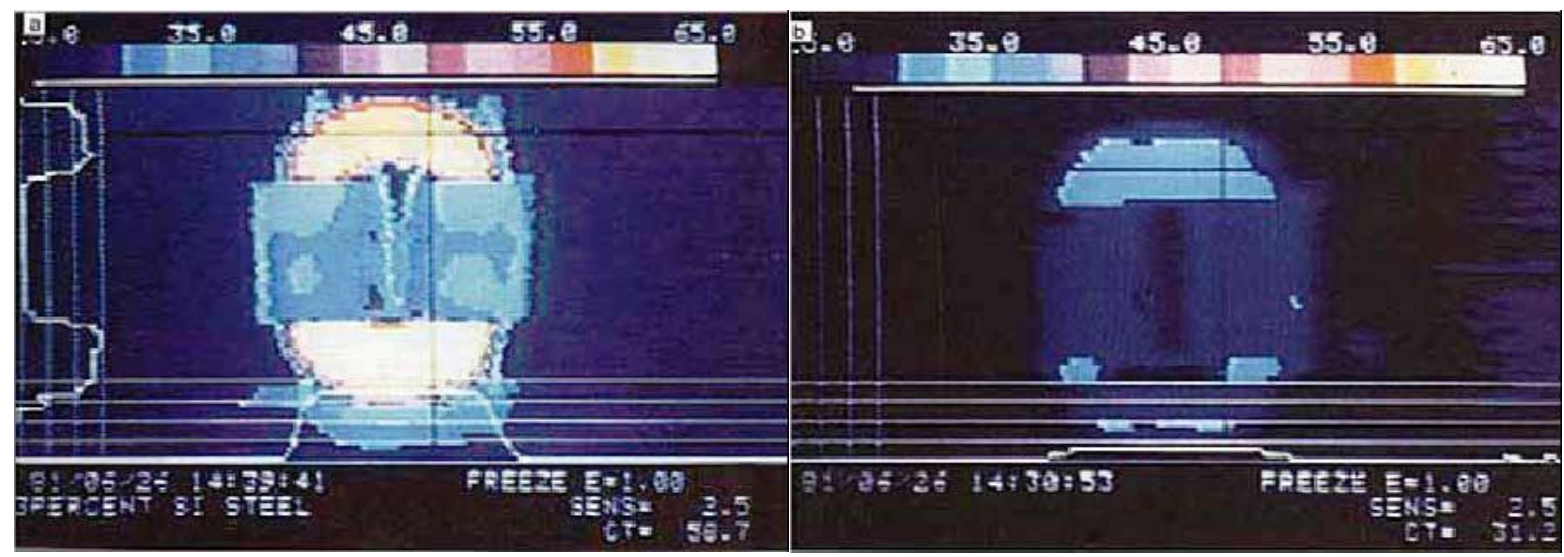

Figura 1. Imagens termográficas: à esquerda núcleo de $\mathrm{FeSi}$ cristalino à $58,7^{\circ} \mathrm{C}$ e à direita núcleo de liga amorfa à $31,2^{\circ} \mathrm{C}$ ambos com enrolamentos energizados e sem carga [4].

* Contribuição técnica ao 69 Congresso Anual da ABM - Internacional e ao 14ํㅡㄹ ENEMET - Encontro Nacional de Estudantes de Engenharia Metalúrgica, de Materiais e de Minas, 21 a 25 de julho de 2014, São Paulo, SP, Brasil. 


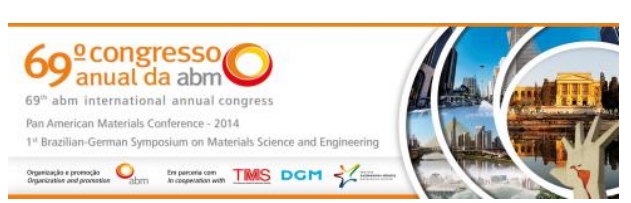

Tal redução das perdas é atribuída à maior resistividade elétrica $(\rho)$, menor espessura $(e)$ da lâmina e menor coercividade $\left(H_{c}\right)$ do material amorfo. Entretanto, devido ao menor valor de indução de saturação $\left(B_{m}\right)$ e menor fator de empacotamento $\left(k_{s}\right)$ da lâmina de liga amorfa, a massa do transformador tende a ser maior que seu similar de mesma potência com núcleo de FeSi cristalino [5]. Tal aumento de massa é totalmente indesejado pela indústria aeronáutica.

Pode-se observar na Tabela 1 as características típicas de ambos os materiais [6] e pelos símbolos pode-se notar as características indesejáveis (*) ou desejáveis (:) na liga FeBSi amorfo em relação ao FeSi cristalino, para aplicação em transformadores utilizados em aeronaves.

Tabela 1. Comparativo de características de ambos os materiais

\begin{tabular}{lcc}
\multicolumn{1}{c}{ Características } & $\begin{array}{c}\text { FeBSi } \\
\text { amorfo }\end{array}$ & $\begin{array}{c}\text { FeSi } \\
\text { Cristalino }\end{array}$ \\
\hline : Indução de saturação $\left(B_{m}\right)$ & $1,56 \mathrm{~T}$ & $2,03 \mathrm{~T}$ \\
\hline :) Coercividade $\left(H_{c}\right)$ & $<4 \mathrm{~A} / \mathrm{m}$ & $<30 \mathrm{~A} / \mathrm{m}$ \\
\hline : Fator de empilhamento $\left(k_{s}\right)$ & 0,84 & 0,95 \\
\hline :) Resistividade elétrica $(\rho)$ & $130 \mu \Omega . \mathrm{cm}$ & $45 \mu \Omega . \mathrm{cm}$ \\
\hline :) Espessura da lâmina $(e)$ & $30 \mu \mathrm{m}$ & $300 \mu \mathrm{m}$ \\
\hline :) Densidade $(d)$ & $7,18 \mathrm{~g} / \mathrm{cm}^{3}$ & $7,65 \mathrm{~g} / \mathrm{cm}^{3}$ \\
\hline Fonte: Adaptado de $[6]$. & &
\end{tabular}

As características da lâmina de metal amorfo são obtidas pelo seu processo de produção que consiste no resfriamento extremamente rápido da liga a partir de sua fase líquida. Ligas eutéticas são caracterizadas por temperaturas de fusão relativamente baixas, na ordem de $1200^{\circ} \mathrm{C}$, que permitem altas taxas de resfriamento, entre $10^{5}$ e $10^{6 \circ} \mathrm{C} / \mathrm{s}$, limitando assim o ordenamento cristalino dos átomos na fase sólida [7]. Pelo menos uma dimensão, do material resfriado, deve ser pequena para que o calor possa ser eficientemente extraído, tipicamente tal dimensão está na faixa de 25 até $50 \mu \mathrm{m}$ [8]. A Figura 2 mostra de forma simplificada o processo para fabricação da lâmina de liga amorfa.

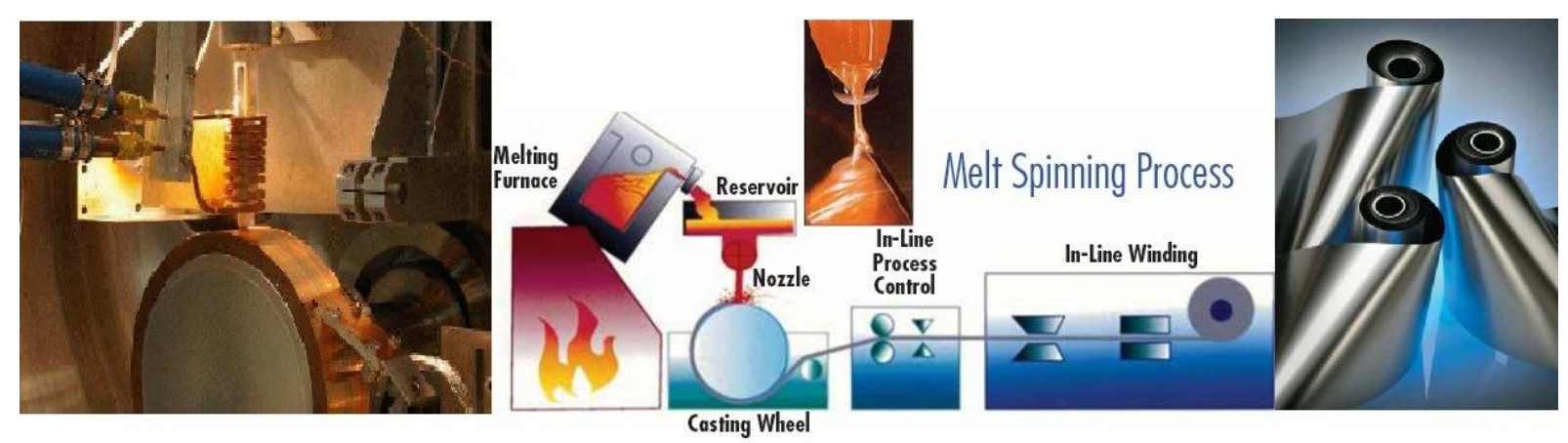

Figura 2. Processo simplificado de fabricação da lâmina de liga amorfa. Fonte: Adaptado de [8-10]

Não existe dúvida que transformadores elétricos são muito convenientes em circuitos elétricos, mas seus tamanhos e pesos são pontos que desfavorecem o uso destes em aeronaves. Quando usados, eles devem ser altamente eficientes e confiáveis em todas as condições de trabalho [11]. O material do núcleo é selecionado para operar com altos valores de indução, mas sem chegar ao ponto de saturação [12], pois um transformador, operando com valores de indução muito próximos ao valor de saturação, pode se autodestruir por excesso de corrente

* Contribuição técnica ao $69^{\circ}$ Congresso Anual da ABM - Internacional e ao 14ํㅡㄹ ENEMET - Encontro Nacional de Estudantes de Engenharia Metalúrgica, de Materiais e de Minas, 21 a 25 de julho de 2014, São Paulo, SP, Brasil. 


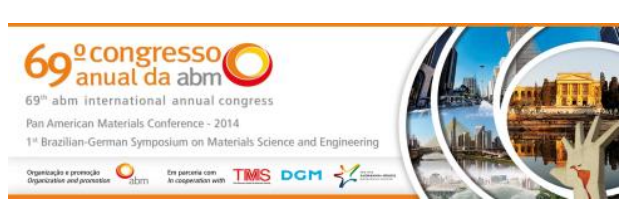

elétrica se houver elevação de tensão na sua alimentação, não acompanhada de elevação da indução no núcleo.

Em razão da confiabilidade, pode-se projetar o transformador para operação em valores menores de indução, especialmente na faixa denominada "joelho" da curva $B H$ de magnetização inicial do material usado no núcleo [13], determinando assim um valor $B_{j}$ de indução a ser usado no lugar de $B_{m}$.

Utilizando-se dados disponibilizados por fabricantes e dados calculados, deseja-se encontrar os valores adequados de indução de cada material e, assim, projetar dois transformadores de mesma potência, mesmas tensões de entrada e saída na frequência de $400 \mathrm{~Hz}$ e calcular respectivamente suas massas e perdas para comparação.

\section{MATERIAIS E MÉTODOS}

A escolha de materiais comercialmente disponíveis que representem a classe de ligas amorfas e a classe de FeSi cristalino foi limitada pela disponibilidade de dados de suas características operando em $400 \mathrm{~Hz}$, portanto a existência de outros materiais com melhor performance e características superiores devem ser consideradas.

Para esta análise foram escolhidas duas ligas: (i) ligas FeBSi amorfas 2605SA1 produzido pela Metglas [14-16] e (ii) FeSi cristalino Microsil .004" produzido pela Magnetic Metals [17].

A falta da curva $B H$ de magnetização inicial para o 2605SA1 nos documentos disponibilizados pelo fabricante Metglas gerou a necessidade de se modelar matematicamente o laço de histerese presente no data sheet [15] pertinente ao núcleo para transformador monofásico. Desta forma, utilizou-se o método descrito por Wlodarski [18] que ajusta uma função $B(H)$ cotangente hiperbólica à curva do laço de histerese experimental a partir da determinação de 5 variáveis: $M a, M b, a, b$ e $c$ ajustadas pelo solver de método GRG Não Linear de uma planilha eletrônica Excel. Para plotar a curva $B H$ de magnetização inicial utiliza-se outra função $B(H)$ que não necessita do parâmetro $c$. A partir destas informações obteve-se um gráfico $\mathrm{BH}$ em escalas lineares.

A curva de magnetização inicial para o material Microsil .004", disponibilizada pelo fabricante, apresenta-se em escalas logarítmicas que dificulta a determinação da região de "joelho" da curva $B H$, portanto construiu-se um gráfico em planilha eletrônica Excel de forma a obter a mesma curva em escalas lineares.

Para o projeto de cada transformador utilizou-se a equação da Lei de Indução de Faraday modificada para aplicação dos parâmetros específicos de cada material usado no núcleo, conforme mostrado na Equação 1.

$$
P_{o}=4,44 f N_{2}\left(\delta A_{c}\right)\left(B_{j} D C k_{s}\right) 10^{-4}
$$

As variáveis $D$ e $C$ representam os lados da área magnética bruta do núcleo, mostrado na Figura 3 , as variáveis $A_{c}$ e $\delta$ representam respectivamente a área transversal do fio de cobre e a densidade de corrente, esta última relacionada à capacidade de dissipação de calor do transformador [19], enquanto $N_{2}$ representa a quantidade de espiras do enrolamento secundário do transformador. A potência de saída, $P_{o}$, e a frequência de operação, $f$, são as mesmas para ambos os projetos de transformadores.

\footnotetext{
* Contribuição técnica ao $69^{\circ}$ Congresso Anual da ABM - Internacional e ao 14ํㅡㄹ ENEMET - Encontro Nacional de Estudantes de Engenharia Metalúrgica, de Materiais e de Minas, 21 a 25 de julho de 2014, São Paulo, SP, Brasil.
} 

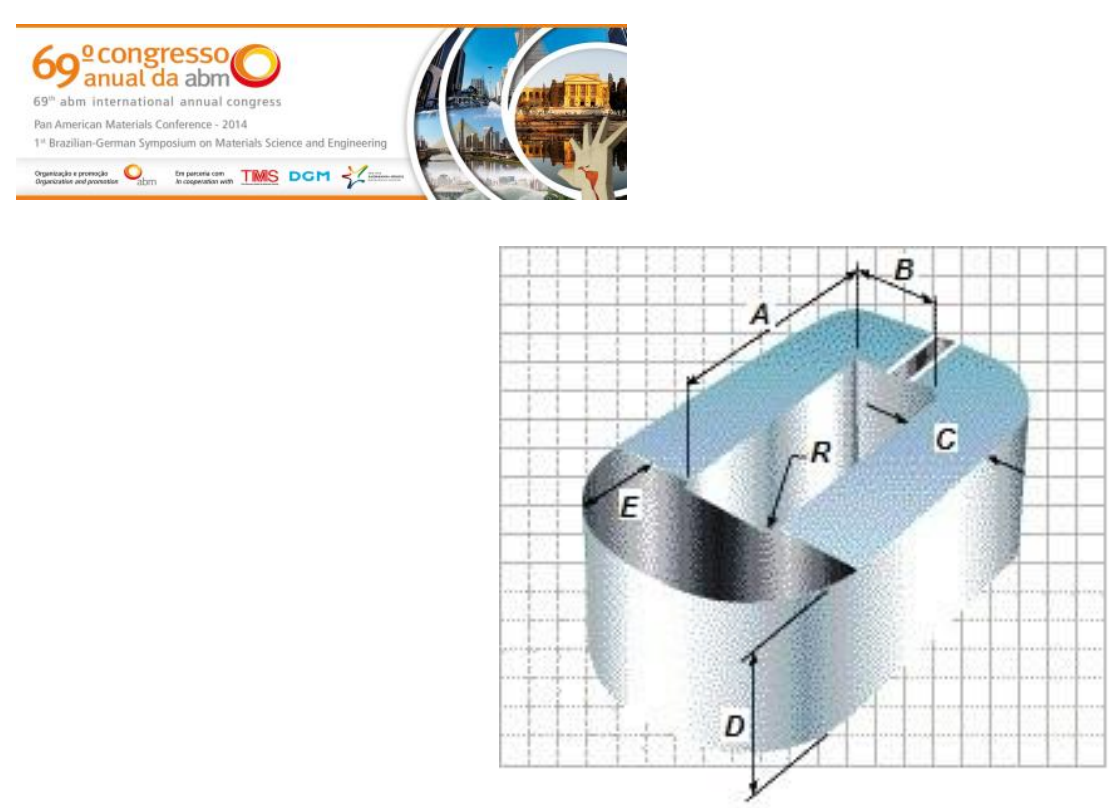

Figura 3. Dimensionamento típico para os núcleos abordados neste estudo. Fonte: Adaptado de [20]

Observando-se a Equação 1, nota-se que existem vários valores possíveis de $C, D$ e $N_{2}$, para obter um mesmo valor de $P_{o}$, entretanto utilizou-se planilha eletrônica Excel com a ferramenta de análise Solver de modo a encontrar valores $C, D$ e $N_{2}$, que minimizem a massa total do transformador, com as seguintes restrições: $C=D \neq 0$, $N_{2}$ inteiro $\neq 0$.

A área da "janela", formada pelas dimensões $A$ e $B$, está relacionada à soma das áreas transversais de todas as espiras dos enrolamentos primário e secundário dividida por um fator $k_{u}$ de preenchimento, mais uma vez utilizou-se restrição no Solver de modo que $A=B \neq 0$, obtendo-se assim uma "janela" no formato quadrado. A massa do núcleo é obtida pelo uso da Equação 2.

$$
\text { Massa do núcleo }=k_{s} \cdot d \cdot(2 \cdot A \cdot C \cdot D)+(2 \cdot B \cdot C \cdot D)+\left(3,14 \cdot C^{2} \cdot D\right) / 1000
$$

A massa total de cobre é calculada somando as áreas transversais de cada espira e multiplicando o resultado pelo comprimento da espira média $(\mathrm{Im})$ multiplicado pela densidade do cobre de valor $8,9 \mathrm{~g} / \mathrm{cm}^{3}$.

\section{RESULTADOS E DISCUSSÃO}

A partir do laço de histerese de um núcleo feito da lâmina 2605SA1, tratado termicamente, envolto em campo magnético longitudinal [15], foram extraídos pontos os quais serviram de referência para a modelagem matemática [18], visando minimizar as diferenças entre pontos calculados e pontos do laço de histerese através do ajuste de 5 variáveis mostradas na Tabela 2, seguindo as seguintes restrições: $M b>M a ; 10<a<200 ; 0,1<b<2 ; 0,1<c<2 ; M a+M b=1241409 \mathrm{~A} / \mathrm{m}$

Tabela 2. Variáveis de ajuste da função $B(H)$ para 2605SA1

\begin{tabular}{cc}
\hline Variável & Valor Ajustado \\
\hline$M a$ & $184848 \mathrm{~A} / \mathrm{m}$ \\
\hline$M b$ & $1056561 \mathrm{~A} / \mathrm{m}$ \\
\hline$a$ & $16,692 \mathrm{~A} / \mathrm{m}$ \\
\hline$b$ & $0,429 \mathrm{~A} / \mathrm{m}$ \\
\hline$c$ & $1,423 \mathrm{~A} / \mathrm{m}$ \\
\hline
\end{tabular}

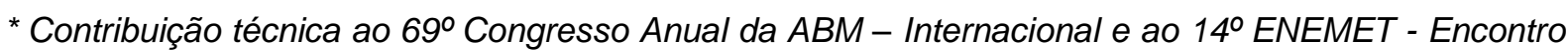
Nacional de Estudantes de Engenharia Metalúrgica, de Materiais e de Minas, 21 a 25 de julho de 2014, São Paulo, SP, Brasil.
} 
Com exceção da variável $c$, as demais são suficientes para obter a curva de magnetização inicial calculada para a lâmina 2605SA1, conforme mostrado na Figura 4.

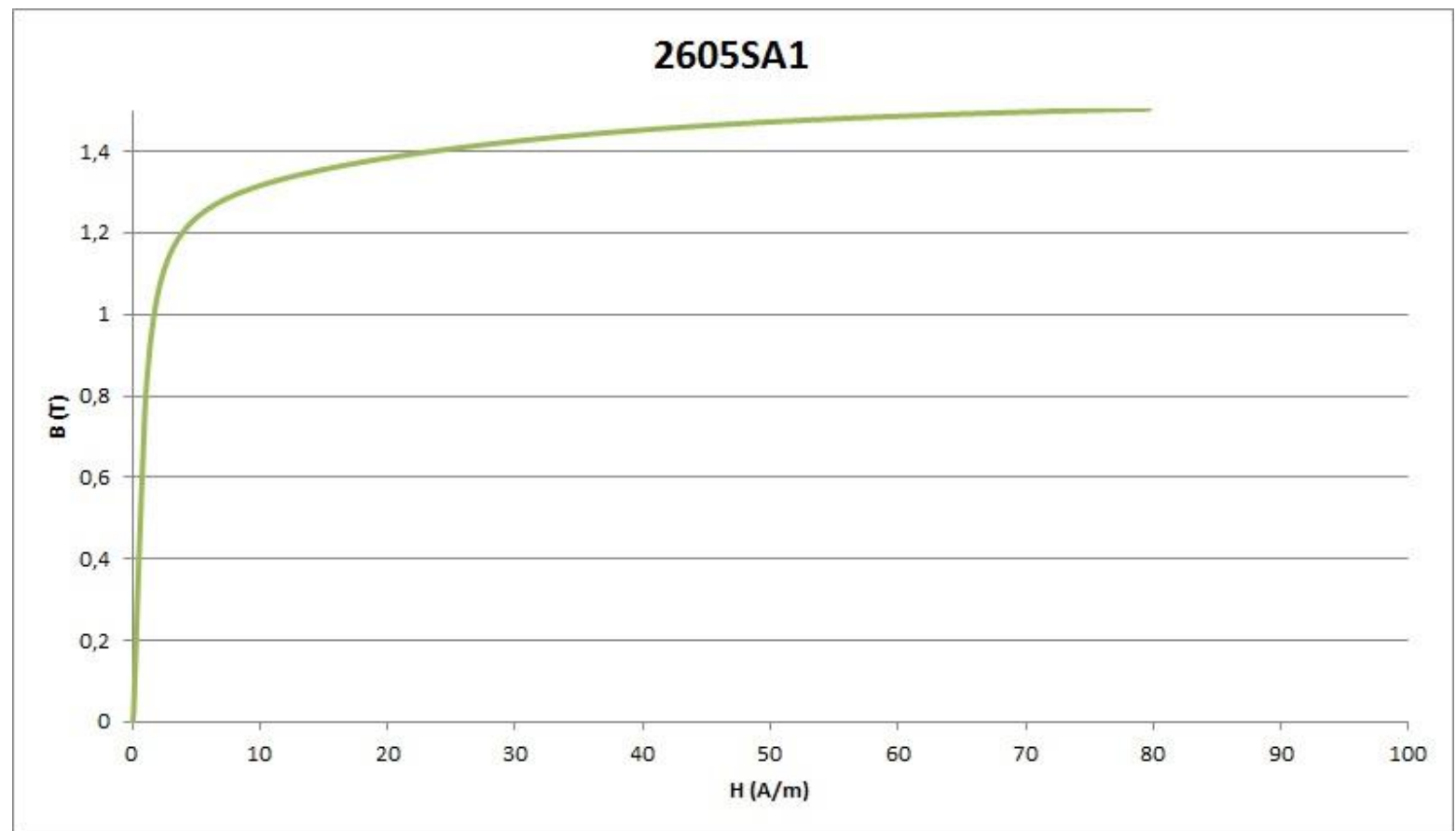

Figura 4. Curva calculada de magnetização inicial da lâmina 2605SA1.

A curva de magnetização inicial da lâmina Microsil .004" originalmente em escala logarítmica [17] foi plotada em escala linear, conforme mostrado na Figura 5, de modo a permitir a comparação com a curva apresentada na Figura 4.

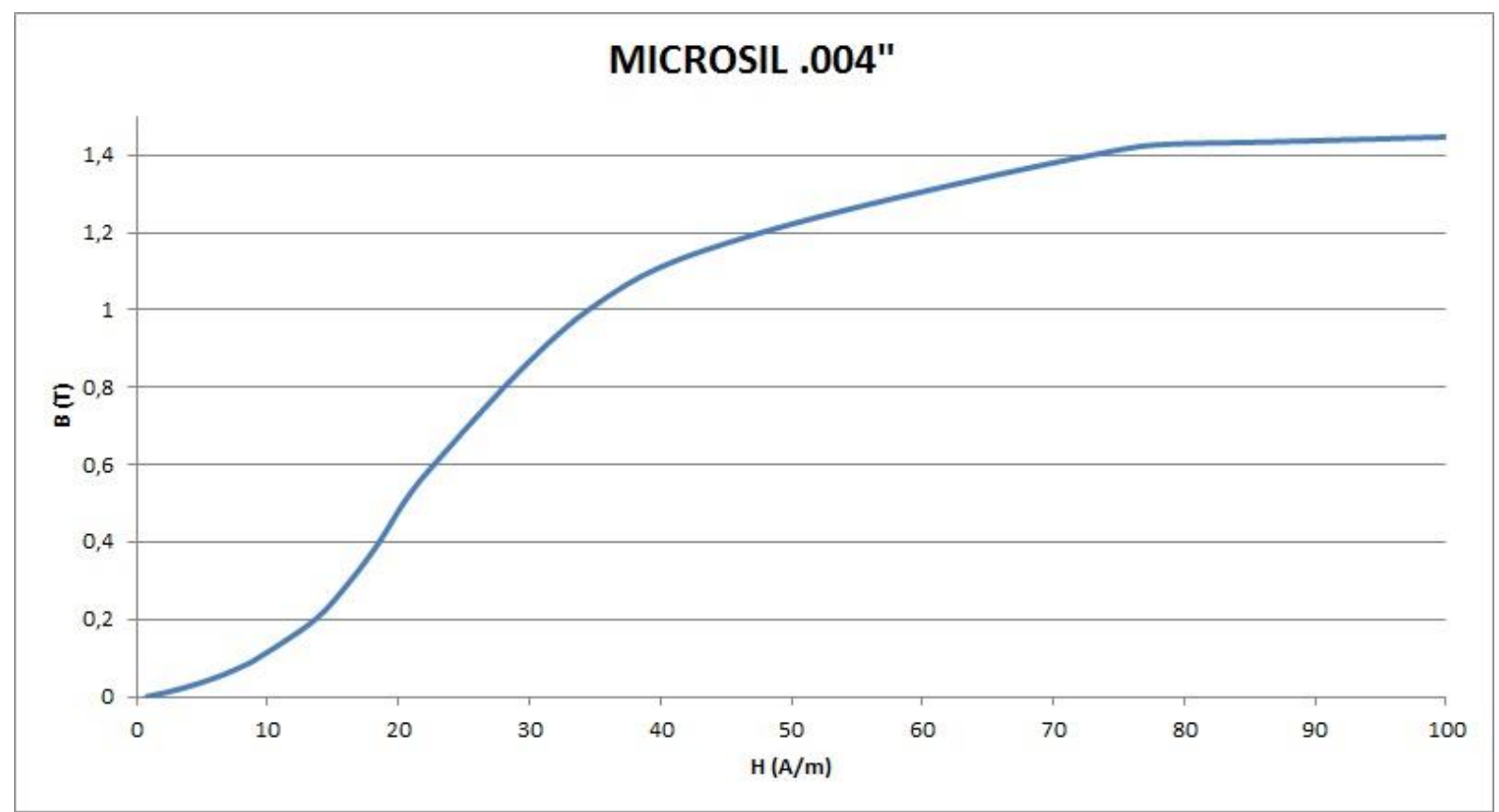

Figura 5. Curva de magnetização inicial da lâmina Microsil .004".

\footnotetext{
* Contribuição técnica ao $69^{\circ}$ Congresso Anual da ABM - Internacional e ao 14ํㅡㄹ ENEMET - Encontro Nacional de Estudantes de Engenharia Metalúrgica, de Materiais e de Minas, 21 a 25 de julho de 2014, São Paulo, SP, Brasil.
} 


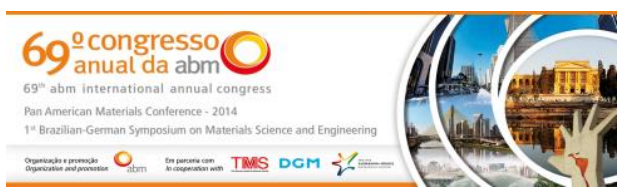

Observando-se ambas as curvas e cada comportamento da indução $(B)$ mediante o campo magnético externo $(H)$, optou-se adotar um valor de $B_{j}=1,1 \mathrm{~T}$ para o material 2605SA1 e um valor de $B_{j}=1,2 \mathrm{~T}$ para o material Microsil .004".

A Tabela 3 mostra todos os parâmetros iniciais dos projetos dos transformadores de ambos os materiais, observando que o valor de densidade de corrente $(\delta)$ tabelado pela potência de saída do transformador [19] foi dobrado, conforme recomendação, por ser um projeto de transformador operando em $400 \mathrm{~Hz}$ [21]. O fator de empilhamento $\left(k_{s}\right)$ listado na Tabela 3 é maior que o mencionado no "data sheet" do fabricante [15], pois a liga amorfa 2605SA1 era comercializada anteriormente sob o código 2605S-2 [9], havendo estudos que indicam um fator de empilhamento $\left(k_{s}\right)$ variando de 0,88 até 0,92 [7,22] para o material $2695 \mathrm{~S}-2$ desde que devidamente processado.

Tabela 3. Dados desejados e calculados para ambos os projetos

\begin{tabular}{ccc}
\hline Parâmetro & $2605 \mathrm{SA} 1$ & Microsil .004" \\
\hline Tensão de entrada $\left(V_{\text {in }}\right)$ & $28 \mathrm{~V}$ & $28 \mathrm{~V}$ \\
\hline Tensão de saída $\left(V_{\text {out }}\right)$ & $28 \mathrm{~V}$ & $28 \mathrm{~V}$ \\
\hline Potência de saída $\left(P_{o}\right)$ & $1000 \mathrm{VA}$ & $1000 \mathrm{VA}$ \\
\hline Densidade de corrente $(\delta)$ & $5 \mathrm{~A} / \mathrm{mm}^{2}$ & $5 \mathrm{~A} / \mathrm{mm}^{2}$ \\
\hline Seção transversal do fio $\left(A_{c}\right)$ & $7,14 \mathrm{~mm}^{2}$ & $7,14 \mathrm{~mm}^{2}$ \\
\hline Frequência de operação $(f)$ & $400 \mathrm{~Hz}$ & $400 \mathrm{~Hz}$ \\
\hline Fator de preenchimento $\left(k_{u}\right)$ & 0,785 & 0,785 \\
\hline Fator de empilhamento $\left(k_{s}\right)$ & 0,88 & 0,90 \\
\hline Indução $\left(B_{j}\right)$ & $1,1 \mathrm{~T}$ & $1,2 \mathrm{~T}$ \\
\hline Espiras no primário $\left(N_{1}\right)$ & 35 & 34 \\
\hline Espiras no secundário $\left(N_{2}\right)$ & 35 & 34 \\
\hline Dimensões do núcleo $(C=D)$ & $2,16 \mathrm{~cm}$ & $2,07 \mathrm{~cm}$ \\
\hline Dimensões do núcleo $(A=B)$ & $2,52 \mathrm{~cm}$ & $2,49 \mathrm{~cm}$ \\
\hline Densidade do material $(d)$ & $7,18 \mathrm{~g} / \mathrm{cm}{ }^{3}$ & $7,65 \mathrm{~g} / \mathrm{cm}$ \\
\hline Comprimento espira média $(I m)$ & $12,59 \mathrm{~cm}$ & $12,19 \mathrm{~cm}$ \\
\hline Perdas do material em $400 \mathrm{~Hz}$ & $1,6 \mathrm{~W} / \mathrm{kg}$ & $6,0 \mathrm{~W} / \mathrm{kg}$ \\
\hline Massa do núcleo & $0,49 \mathrm{~kg}$ & $0,48 \mathrm{~kg}$ \\
\hline Perda no núcleo & $0,79 \mathrm{~W}$ & $2,92 \mathrm{~W}$ \\
\hline Massa de cobre das espiras & $0,56 \mathrm{~kg}$ & $0,53 \mathrm{~kg}$ \\
\hline Massa total do transformador & $1,06 \mathrm{~kg}$ & $1,01 \mathrm{~kg}$ \\
\hline Perda no cobre em $75^{\circ} \mathrm{C}$ & $0,95 \mathrm{~W}$ & $0,90 \mathrm{~W}$ \\
\hline Perda total no transformador & $1,74 \mathrm{~W}$ & $3,81 \mathrm{~W}$
\end{tabular}

Apesar da grande redução de perdas no núcleo do transformador de liga amorfa, há o inconveniente do mesmo ter massa total maior que seu similar em FeSi cristalino de mesma potência.

Alguns autores [23] observam que um núcleo de liga amorfa operando em menor temperatura, permite maior dissipação de calor dos enrolamentos de cobre, portanto pode-se diminuir a seção transversal do fio de modo a diminuir a massa total do transformador.

Considerando-se a temperatura do núcleo de liga amorfa informada na Figura 1 e que a resistividade do fio de cobre para transformadores a $75^{\circ} \mathrm{C}$ é de $0,0216 \Omega / \mathrm{m} / \mathrm{mm}^{2}$, então se pode calcular a resistência elétrica total dos enrolamentos a $75^{\circ} \mathrm{C}(T 1)$ e recalcular uma seção transversal menor de fio que apresente a mesma resistência elétrica em $35^{\circ} \mathrm{C}$, utilizando-se a Equação 3.

\footnotetext{
* Contribuição técnica ao 69ำ Congresso Anual da ABM - Internacional e ao 14ํㅡㄹ ENEMET - Encontro Nacional de Estudantes de Engenharia Metalúrgica, de Materiais e de Minas, 21 a 25 de julho de 2014, São Paulo, SP, Brasil.
} 


$$
R \Omega_{T 2}=R \Omega_{T 1} \cdot(234,5+T 2) /(234,5+T 1)
$$

O novo valor da resistência ôhmica dos enrolamentos na temperatura T2 é fornecido pela variável $R \Omega$ T2.

Tal procedimento ocasiona a diminuição das dimensões $A$ e $B$ do núcleo, a redução do comprimento da espira média $(\mathrm{Im})$ do enrolamento de cobre, a redução da massa de cobre dos enrolamentos e redução de massa do núcleo de liga amorfa, conforme mostrado na Tabela 4.

Tabela 4. Dados recalculados para transformador com núcleo de material 2605SA1

\begin{tabular}{cc}
\hline Parâmetro & Valor \\
\hline Resistência total dos enrolamentos em $75^{\circ} \mathrm{C}\left(R \Omega_{T 1}\right)$ & $0,027 \Omega$ \\
\hline Seção transversal do fio recalculada $\left(A_{C}\right)$ & $6,22 \mathrm{~mm}^{2}$ \\
\hline Dimensões do núcleo recalculadas $(A=B)$ & $2,36 \mathrm{~cm}$ \\
\hline Massa do núcleo recalculada & $0,48 \mathrm{~kg}$ \\
\hline Comprimento espira média recalculada $(I \mathrm{~m})$ & $12,33 \mathrm{~cm}$ \\
\hline Massa total de cobre & $0,48 \mathrm{~kg}$ \\
\hline Massa total recalculada & $0,95 \mathrm{~kg}$ \\
\hline Perda no núcleo recalculada & $0,76 \mathrm{~W}$ \\
\hline Perda nos enrolamentos de cobre recalculada & $0,93 \mathrm{~W}$ \\
\hline Perda total, no transformador, recalculada & $1,69 \mathrm{~W}$ \\
\hline
\end{tabular}

\section{CONCLUSÃo}

O redimensionamento da seção transversal do fio de cobre foi suficiente para oferecer um transformador com núcleo de liga amorfa 2605SA1 de menor perda e de massa total menor que seu equivalente, em potência, feito com núcleo de Microsil .004", embora existam outros requisitos a serem satisfeitos para a homologação do uso aeronáutico [24] de transformadores com núcleo de liga amorfa, o requisito massa não é um fator limitante para este uso.

\section{Agradecimentos}

Ao Programa de Pós Graduação em Ciência e Engenharia de Materiais (PPGCEM) da Universidade Federal de São Carlos (UFSCar) pelo incentivo e suporte à participação de seus alunos neste congresso.

\section{REFERÊNCIAS}

1 Hale J. Boeing 787 from ground up. AERO Magazine. 2006 [acesso em 08 mar. 2014]; 04: 17-23. Disponível em:

www.boeing.com/commercial/aeromagazine/articles/qtr_4_06/AERO_Q406_article4.pdf

2 Sinnett M. 787 no bleed system. AERO magazine. 2007 [acesso em 08 mar. 2014]; 04: 06-11. Disponível em:

www.boeing.com/commercial/aeromagazine/articles/qtr_4_07/AERO_Q407_article2.pdf

3 Raymer, D.P. Aircraft design: a conceptual approach. Reston: AIAA, 1999.

4 Metglas Power: Brochura. 2012 [acesso em 09 mar. 2014]. Disponível em: www.metglas.com/assets/pdf/metglas_power_brochure.pdf

5 Hitachi Amorphous Transformers: Catálogo. 2010 [acesso em 09 mar. 2014].

Disponível em: www.hitachi-ies.co.jp/english/catalog_library/pdf/transformers.pdf

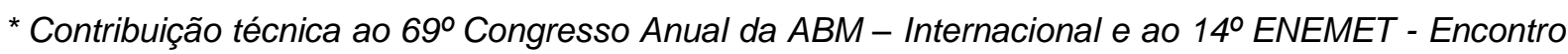
Nacional de Estudantes de Engenharia Metalúrgica, de Materiais e de Minas, 21 a 25 de julho de 2014, São Paulo, SP, Brasil.
} 


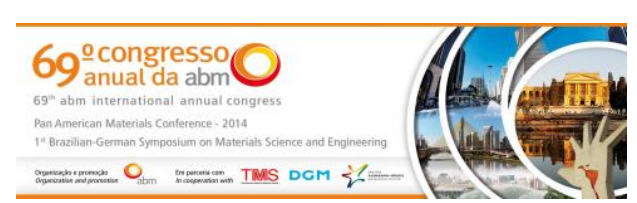

6 Rubicon Metals: Amorphous Ribbons specifications. 2011 [acesso em 09 mar.2014]. Disponível em: http://rubiconmetals.com/wp-content/uploads/2011/07/AmorphousC.pdf

7 Allia P, Soardo GP, Vinai F. Applied research on amorphous magnetic materials. Hyperfine Interactions. 1989;45:35-53.

8 Decristofaro N. Amorphous metals in electric power distribution applications. MRS Bulletin. 1998; 23(5):50-56.

9 Mouhamad M. Reduction des pertes a vide des transformateurs de distribution par utilisation de rubans amorphes [these de doctorat]. Cachan: L' Ecole Normale Superieure de Cachan; 2012.

10 Metglas Amorphous Brazing Foil: Brochura. 2012 [acesso em 09 mar. 2014]. Disponível em: http://www.metglas.com/assets/pdf/2012_metglas_brazing_brochure.pdf

11 Morris AL. Small Power Transformers for Aircraft Electrical Equipments. Proceedings of the IEE Part III: Radio Communication Engineering. 1949;96(42):279-288.

12 Kiltie O. Aircraft transformers - Small and light. Transactions of the American Institute of Electrical Engineers. 1943;62(12):899-902.

13 Flanagan WM. Handbook of transformer design and applications. New York: McGraw Hill, 1992.

14 Metglas Magnetic Alloy 2605SA1: Technical Bulletin. 2009 [acesso em 10 mar. 2014]. Disponível em: www.rotima.ch/fileadmin/downloads/Amorphe_Materialien/2605SA1_techn__bulletin.pdf

15 Metglas Amorphous Alloys for Transformer Cores: Data Sheet. 2011 [acesso em 10 mar 2014]. Disponível em: www.metglas.com/assets/pdf/2605sa1.pdf

16 Metglas 2605SA1 Iron Based Alloy: Material Safety Data Sheet. 2013 [acesso em 10 mar. 2014] Disponível em: www.metglas.com/assets/pdf/msds/msds2605sa1.pdf

17 Magnetic Metals: Tape Wound Core Design Manual. 2005 [acesso em 10 mar. 2014]. Disponível em: www.magmet.com/tapewound/tapewound.pdf

18 Wlodarski Z. Analytical description of magnetization curves. Physica B. 2006; 373:323327.

19 Martignoni A. Transformadores. Porto Alegre: Globo S.A.,1971.

20 Transformer core design [página da internet]. Conway: [acesso em 20 nov. 2013]. Disponível em: www.metglas.com/products/metglaspower/transformer_core_design.asp

21 McLyman TWM. Transformer and Inductor Design Handbook. New York: Marcel Dekker, 2004.

22 Boglietti A, Ferraris P, Lazzari M, Profumo F. Design and Realization of Amorphous Materials Transformers. J. Mater. Eng. 1991;13(2):151-160.

23 Raskin D, Smith $\mathrm{CH}$. Applications of amorphous metals: progress and prospects. In: Luborsky FE. Amorphous metallic alloys. London: Butterworth; 1983. p. 381-400

24 RTCA/DO-160E: Environmental Conditions and Test Procedures for Airborne Equipment. Washington: Radio Technical Commission for Aeronautics Inc; 2004.

\footnotetext{
* Contribuição técnica ao 69 Congresso Anual da ABM - Internacional e ao 14ํㅡㄹ ENEMET - Encontro Nacional de Estudantes de Engenharia Metalúrgica, de Materiais e de Minas, 21 a 25 de julho de 2014, São Paulo, SP, Brasil.
} 